\title{
Géocarrefour
}

Numéro Vol. 85/2 (2010)

Services urbains en réforme dans le monde arabe

Bénédicte Florin

\section{Réforme de la gestion des déchets et reconfigurations des territoires professionnels des chiffonniers du Caire}

\begin{abstract}
Avertissement
Le contenu de ce site relève de la législation française sur la propriété intellectuelle et est la propriété exclusive de l'éditeur.

Les œuvres figurant sur ce site peuvent être consultées et reproduites sur un support papier ou numérique sous réserve qu'elles soient strictement réservées à un usage soit personnel, soit scientifique ou pédagogique excluant toute exploitation commerciale. La reproduction devra obligatoirement mentionner l'éditeur, le nom de la revue, l'auteur et la référence du document.

Toute autre reproduction est interdite sauf accord préalable de l'éditeur, en dehors des cas prévus par la législation en vigueur en France.
\end{abstract}

\section{revues.org}

Revues.org est un portail de revues en sciences humaines et sociales développé par le CLEO, Centre pour l'édition électronique ouverte (CNRS, EHESS, UP, UAPV).

\section{Référence électronique}

Bénédicte Florin, « Réforme de la gestion des déchets et reconfigurations des territoires professionnels des chiffonniers du Caire », Géocarrefour [En ligne], Vol. 85/2 | 2010, mis en ligne le 10 octobre 2010. URL : http:// geocarrefour.revues.org/index7812.html

$\mathrm{DOI}$ : en cours d'attribution

Éditeur : Association des amis de la Revue de Géographie de Lyon

http://geocarrefour.revues.org

http://www.revues.org

Document accessible en ligne à l'adresse suivante : http://geocarrefour.revues.org/index7812.html Ce document est le fac-similé de l'édition papier.

Cet article a été téléchargé sur le portail Cairn (http://www.cairn.info)

\section{CAIR N}

Chercher. Repérer. Avancer.

Distribution électronique Cairn pour Association des amis de la Revue de Géographie de Lyon et pour Revues.org (Centre pour l'édition électronique ouverte)

(c) Géocarrefour 


\section{Bénédicte FLORIN}

Université de Tours, UMR 6173 CNRS, CITERES -

EMAM, Equipe Monde arabe et Méditerrané

\section{MOTS CLÉS}

Réforme, déchets, chiffonniers, organisation professionnelle, territoire, stratégie, ajustement, Le Caire, Egypte.

\section{KEY WORDS}

Reform, solid waste, ragand-bone men, professional organisation, territory, strategy, adjustment, Cairo, Egypt.

\section{1 - Le terme zabbâlin (sing} zabbâl, dérivé de zabbal, la poubelle, le déchet) que l'on prononce zabaline, se traduit par " chiffonnier", bien que ce mot soit réducteur de la diversité des façons de travailler, des activités et des positions des zabbâlin, comme on le verra par la suite.

\section{Réforme de la gestion des déchets et reconfigurations des territoires professionnels des chiffonniers du Caire}

RÉSUMÉ

Au Caire, depuis les années 2000, le système informel traditionnel de collecte et recyclage des déchets, celui des chiffonniers, les zabbâlin, a été totalement bouleversé par la délégation de la gestion à des sociétés privées. Après avoir présenté l'organisation professionnelle des ramasseurs et recycleurs composant la communauté des chiffonniers avant la " privatisation ", cet article examine les stratégies d'ajustement à la réforme qu'ils mettent en œuvre : ces pratiques reconfigurent la structure et les relations internes à la communauté mais créent aussi de nouveaux territoires professionnels, fondés sur des temporalités et réseaux de travail inédits. Si quelques chiffonniers ont réussi à intégrer le système formel, si d'autres ont signé des contrats de soustraitance, nombreux sont ceux qui ont initié des façons inédites de pratiquer de leur activité, basculant dans l'illégalité.

\section{ABSTRACT}

In Cairo, with the arrival of private companies, the informal system of solid waste management, rag-and-bone men, called "zabbâlin", has been totally transformed since 2000. Our paper will provide a brief overview of how informal garbage collectors used to operate before the "privatization" and examine the actors' strategies of adjustment given the new context. These new practices reconfigure deeply the structure and organization of the zabbalîn community : it creates new professional territories based on different activities. Some of the zabbâlin have been incorporated into the formal labor sector and others have signed contracts with private companies to recycle waste. But most of them have resorted to other forms of organization, often illegal.

Au Caire, jusqu'aux années 2000, I'organisation de la collecte et du recyclage des déchets solides ne relevait pas d'un service public, à la différence de la distribution de l'eau, de l'électricité ou de I'assainissement. Certes, les services municipaux de propreté de la capitale entretenaient les espaces publics mais, depuis le ramassage des ordures jusqu'à la revente des produits finis ou semi-finis après recyclage, le système de gestion des déchets était le fait des chiffonniers, les zabbâlin', qui forment une véritable corporation dont quasiment tous les membres sont liés, directement ou indirectement, à cette activité. En germe depuis les années 1990, la délégation de ce service à des entreprises européennes et égyptiennes a pris effet à partir de 2002 au Caire, à la suite d'Alexandrie où l'entreprise Véolia gère la collecte depuis 2000. Qualifiée de "privatisation" - terme impropre puisqu'il n'existait pas auparavant de service public de collecte - la délégation de service provoque une crise mettant en jeu des acteurs aussi différents que les Cairotes, les autorités publiques, les médias, les représentants des sociétés européennes et ceux des associations de défense des zabbâlin, ainsi que ces derniers qui, sans en avoir été avertis préalablement, n'ont plus accès aux déchets. Les stratégies d'ajustement et de contournement de la contrainte mises en œuvre par les zabbâlin redéfinissent leur rôle dans la hiérarchie du métier et reconfigurent leurs territoires professionnels.

\section{L'ORGANISATION PROFESSIONNELLE DES ZABBÂLIN: ACTEURS, CIRCULATIONS ET TERRITOIRES D'UNE CORPORATION}

"Collines de décombres", "vilénies des habitants " et premières initiatives publiques

La littérature scientifique sur Le Caire n'évoque que de façon parcimonieuse et fragmentée
I'histoire des déchets solides dans la ville, alors que sont amplement décrits les systèmes d'adduction d'eau, le tracé viaire ou, pour l'époque moderne, les progrès concernant l'éclairage des rues au gaz puis à l'électricité, ou les formes de gestion des services "publics" associant le plus souvent les compagnies privées concessionnaires pour l'eau, le gaz et l'électricité. $L^{\prime}$ une des premières occurrences connue d'une action publique quant aux ordures est fournie par I'historien Gabartî (1753-1825) qui date de 1798 l'ordre, mû par des motifs sanitaire et prophylactique, " de balayer et d'arroser les rues, $d^{\prime}$ entretenir la propreté de la voie publique en ce qui concerne les rebuts et les ordures" (Raymond, 1993, p. 293). A l'instar de l'éclairage nocturne des rues, obligatoire pour des raisons de sécurité, les autorités françaises de l'Expédition d'Egypte (1798-1801) laissent à l'initiative des riverains la responsabilité de la propreté urbaine. En dépit des amendes, l'ordre semble être suivi de peu d'effet si l'on en croit les descriptions qu'historiens et voyageurs font du Caire, témoignant de l'indescriptible saleté de la ville islamique et plus encore de ses faubourgs constitués en grande partie de bicoques miséreuses. Ainsi, Marcel Clerget explique-t-il, dans sa magistrale Etude de géographie urbaine, que le Khalig - canal - qui traversait la ville fait office de dépotoir :

"Tous les voyageurs européens qui ont vu le Khalig au moment de la crue parlent en termes éloquents du spectacle offert à leurs yeux (...). L'eau croupit jusqu'en avril dit Volney. II y a tant d'immondices dans le Khalig, ajoute Niebuhr, qu'ils ne peuvent sécher et que l'odeur de la boue empeste l'air durant les grandes chaleurs et cause des maladies (...). Thévenot au $17^{\circ}$ siècle et Le Bruyn au $18^{e}$ n'ont pas été moins édifiés. Le premier raconte 'Même avant que le Khalig ait cessé complètement de couler, toutes les ordures 
s'y arrêtent; plus tard, c'est une puanteur horrible à cause des ordures et des vilénies de ceux qui ont des fenêtres au dessus, outre quantité de charognes qu'on y jette (...). L'odeur qui se sent de très loin me fit connaître bientôt ce que c'était et son infection horrible $m^{\prime}$ a souvent fait étonner que cela ne leur donne pas la peste tous les ans " (Clerget, 1934, T. II, p. 6).

Les historiens s'accordent encore sur les collines de décombres qui bornent Le Caire et qui, constituées de ruines et gravats divers, sont des lieux servant de dépotoirs où I'on trouve des charognes ${ }^{2}$ et ordures putrescibles en tout genre: "Comme une plaie affreuse, elles rongèrent insensiblement la bordure des quartiers extérieurs. (...) A l'époque turque, le mal ne fit $q u$ 'empirer, les amas de décombres devinrent de véritables montagnes, on en vit surgir de nouveaux, en particulier à l'ouest de la ville. (...) Lorsque soufflait un vent violent, des nuages d'une poussière aveuglante et malsaine s'étendaient sur Le Caire, s'ajoutant aux nuages de sable venus des déserts limitrophes " (Clerget, 1934, T. II, p. 6).

Le règne de Muhammad 'Ali (1805-1848) marque l'entrée de l'Egypte dans la modernité qui, en ville, se traduit notamment par le contrôle du balayage et de l'arrosage des voies surveillées par des autorités d'arrondissements. C'est sous son autorité que plusieurs collines de décombres sont nivelées - ces " nids d'infection "-, que les étangs putrides sont comblés ainsi que les canaux sont

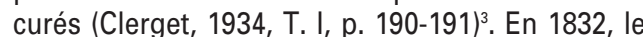
ramassage des déchets semble être, au moins en partie, organisé par les autorités publiques si I'on en croit le voyageur Saint-John:

"Les rues, naguère sales et dégoûtantes, sont maintenant pour la plupart remarquables de propreté, car elles sont balayées trois fois par jour... Les ordures sont réunies en tas, et quatre cents charrettes, traînées par de jeunes bœufs, sont employées pour les déposer hors de la ville" (Raymond, 1993, p. 299).

La deuxième moitié du $\mathrm{XIX}^{\mathrm{e}} \mathrm{s}$. est caractérisée, au Caire ou à Alexandrie, par de spectaculaires progrès en matière d'éclairage public, d'assainissement et d'adduction d'eau (llbert, 1996, 349 ; Raymond, 1993, 308). Si I'œuvre réformatrice du khédive Isma'il (1863-1879) est essentielle en termes de gestion urbaine, par contre, peu d'informations nous sont données concernant spécifiquement les déchets. Cependant, Clerget évoque I'existence d'une société privée chargée du nettoyage des rues dont le contrat est résilié en 1881 en raison de son " incapacité ". En 1898, le Tanzim, organisme responsable de la voirie et de la gestion urbaine, s'intéresse certes au nettoyage, mais $41 \%$ des rues lui échappent encore "faute de fonds, de personnel et de matériel ", sans compter les mauvaises "habitudes des habitants "; en 1901, la proportion passe à $61 \%$ de voies nettoyées quotidiennement et tous les quatre jours pour les autres, tâche effectuée sous le contrôle du Département de I'Hygiène. Clerget précise aussi que ces services de la propreté "avec des moyens de fortune (souvent à la main) et des charrois primitifs " évacuent "une quantité parfois énorme de boue et détritus variés. En 1898, en quatre jours de pluies, 12158 carrioles furent chargées de cette façon (...) une autre fois, on dut emporter 12528 tonnes en quelques jours ". Les services du Tanzim n'évacuent que la moitié des 789 tonnes quotidiennes tandis qu'une partie des déchets restants servent au chauffage de l'eau des bains et aux feux de cuisine. La progression constante du volume d'ordures, supérieur à celui des grandes villes européennes, impose la création de nouveaux dépotoirs qui s'ajoutent au seul site moderne d'incinération, près des abattoirs (Clerget, 1934, T II, p.12-14). En dépit des efforts, liés à la collecte par les premiers camions à partir de 1924, le contraste est immense entre la propreté de la ville européenne promue par le khédive Isma'il où le ramassage est journalier et la vieille ville très irrégulièrement nettoyée et qui l'est surtout lorsque les épidémies menacent (la peste en 1900, le choléra en 1902) :

"La plupart des interventions commanditées par le Service sanitaire (du Tanzim) sont bien des travaux d'édilité ; cependant, elles sont toujours ponctuelles et ne correspondent qu'aux nécessités les plus urgentes sans que la moindre organisation suivant un plan d'ensemble en soit envisagée. La situation est paradoxale. D'une part, le budget du Tanzim ne permet pas l'entretien régulier de la ville, d'autre part, tous les dix ans environ, le Service sanitaire, sous la menace des épidémies, fait nettoyer les quartiers anciens de fond en comble. En 1899, ce sont 175 employés qui ont dû travailler pendant plus de neuf mois au nettoyage de la vieille ville. Cette manière de procéder est d'autant plus étonnante que les chiffres indiquent qu'elle n'est peut-être pas la plus économique " (Arnaud, 1993, p. 260).

Quoi qu'il en soit, les espaces où s'effectue la collecte des déchets sont majoritairement les quartiers de la bourgeoisie cairote et des Européens. Cette fragmentation de la ville, en fonction du statut des quartiers et de leurs habitants, est confirmée par la (relative) propreté qui caractérise la banlieue thermale d'Hélouan (au sud du Caire), les nouveaux quartiers de Choubra (situés au nord) ou encore Héliopolis, ville nouvelle d'initiative privée de la banlieue nord-est où le ramassage des déchets est effectué par la Compagnie du baron Empain, promoteur de la cité ; mais, à partir de 1921, à l'instar des autres services tels que l'éclairage, l'entretien de la voirie, le réseau d'égouts, les services du Tanzim
2 - L'orientaliste Edward Lane (1801-1876) écrit : "All the animals that die in Cairo are cast upon the mounds of rubbish, where they are quickly devoured by vultures, kites and dogs " (Abu-Lughod, 1971, p. 92). Les milans du Caire, oiseaux de proie prolifiques, sont évoqués dès le XVI ${ }^{\mathrm{e}} \mathrm{s}$. par les encyclopédistes et considérés comme contribuant, à leur façon, à la propreté de la ville.

3 - Le Caire n'a alors rien à envier à Paris qui, dans les années 1880, était aussi cerné par des collines d'ordures et d'excréments « dont les germes morbides les plus funestes " étaient apportés par les vents dans la capitale (De Silguy, 1996, p. 29). 
sont, là aussi, responsables de l'évacuation des ordures, "incinérées dans quelques vallons du désert " (Besançon, 1958, p. 133).

Acquise en 1952, l'indépendance ne modifie pas fondamentalement la situation en ce qui concerne la gestion des déchets même si, dès 1949, la municipalité du Caire - baladiyya - est créée et si, de fait, les Egyptiens avaient recouvré la maîtrise de la gestion des services publics dans le cadre du ministère des Affaires rurales et urbaines (Raymond, 1993, p. 346). L'œuvre nationaliste, réformatrice et modernisatrice de Gamal Abdel Nasser (1954-1970) concernera des domaines aussi variés que la nationalisation des services de l'eau et des transports, la construction de logements sociaux ou encore le développement des services publics liés à l'éducation et à la santé. Certes, la loi 38 de 1967, amendée en 1976, réglemente la collecte et la décharge des déchets solides, mais elle n'est pas, semble-t-il, suivie de beaucoup d'effets : en bref, il n'y a pas, avant les années 1980, d'action de grande ampleur sur la gestion des déchets.

\section{L'organisation du système informel avant la "privatisation ": acteurs et compétences professionnelles}

\section{L'évolution du rôle et de la position des wâhiya, les} "oasiens"

L'existence de chiffonniers au Caire est avérée depuis le $X V l^{\text {e }}$ s. et une liste des corporations les associe aux métiers utiles, à l'instar des porteurs d'eau et des portiers; mais, en 1670, ils basculent dans le "groupe des corporations exécrables et impies", les agrégeant aux prostituées, vagabonds, mendiants et voleurs... (Assaad, 1988 , p. 186). Quoi qu'il en soit de ces premiers zabbâlin, à la fin du XIXe s., la collecte des déchets est effectuée par des musulmans, originaires des oasis libyques: les wâhiya (oasiens, sing. wâhî). En échange d'un droit acheté aux propriétaires, ces derniers ont accès aux ordures qu'ils revendent comme combustible. Ils bénéficient aussi des sommes mensuelles versées par les habitants en échange de leur service : jusqu'aux années 1980, "aucune personne en dehors des wâhiya ne peut signer un contrat avec le propriétaire d'un immeuble pour ramasser les ordures, et aussitôt qu'un wâhî acquiert ce droit d'accès, les autres wâhiya ne peuvent plus lui faire concurrence. A la mort d'un wâhî, les immeubles qu'il contrôle passent à ses héritiers. Les wâhiya justifient leur droit d'exclure les zabbâlin des paiements des locataires en faisant référence à une loi coutumière qui leur donne ce droit par héritage. Ils insistent aussi sur la division du travail entre eux et les zabbâlin : ils sont responsables du ramassage des ordures et les zabbâlin de leur transport. Le fait que dans la plupart des cas le zabbâl accomplisse les deux tâches ne semble pas peser lourd (...) Les wâhiya protégeraient leurs droits d'accès contre tout intrus par la violence et le sabotage si nécessaire, mais ils ont rarement recours à ces méthodes car le système est implicitement accepté par toutes les parties concernées, y compris les autorités municipales " (Assaad, 1988, p. 188).

Dans les années 1930, I'installation en ville de migrants coptes, originaires du sud, modifie l'organisation de la collecte : ces derniers, éleveurs de cochons, achètent les déchets organiques aux wâhiya pour nourrir leurs animaux; puis, après négociation et en échange d'une taxe, ils remplacent peu ou prou les wâhiya en collectant eux-mêmes les ordures, par le biais du porte-à-porte. Le ramassage, qui peut parfois compter jusqu'à 300 immeubles, est alors effectué par les hommes, accompagnés de leurs fils, tôt le matin quand le trafic est encore fluide. Les habitants déposent leurs déchets dans des sacs ou des seaux sur le palier de l'escalier principal ou de service et rétribuent mensuellement le wâhî de leur quartier ou parfois directement le "ramasseur ", le zabbâl; le montant de cette rétribution dépend de la "richesse " du quartier. Les déchets, regroupés dans de grands sacs, sont chargés sur des pick-up et transportés jusqu'aux zarâyeb (sing. zerîba), littéralement " porcheries ", où ils sont déversés dans les rues, des cours ou en rez-de-chaussée des habitations afin d'être triés et recyclés. Si les activités économiques de ces quartiers ne se réduisent pas à l'élevage des porcs, loin de là, le terme "zerîba " continue à les qualifier.

De cette organisation découle le fait que, d'une part, chaque famille de zabbâl est liée à un wâhî particulier et travaille dans un îlot spécifique et que, d'autre part, les résidents connaissent leur zabbâl, parfois d'une génération à l'autre. Ce partage territorial repose sur des contrats oraux, fondés sur le principe du 'orf, "ce qui est convenu ".

L'imposition d'un système de licence dans les années 1980 et, davantage encore, la " privatisation " des années 2000 fragiliseront temporairement la position, le rôle et les activités de ces intermédiaires que sont les wâhiya, mais modifieront aussi leurs relations avec les zabbâlin. Ainsi, les réformes marquent-elles, par exemple, la fin de la "taxe" imposée par les premiers aux seconds pour l'accès aux immeubles ou encore des alliances entre les deux corporations ${ }^{5}$ qui tendent à réduire la dépendance ou l'emprise qu'exerçaient les wâhiya sur les zabbâlin (Dollet, 2003, p. 106 ; Desvaux, 2009, p. 61). Ceci ne signifie pas qu'il n'y a plus de hiérarchie professionnelle - bien au contraire ! mais celle-ci n'est plus fondée sur la distinction qui prévalait antérieurement entre les deux activités. 


\section{Encadré : Les sept zarâyeb du Caire}

La zerîba la plus importante en termes de population et d'activités est celle de Manchiat Nasser, enserrée dans le grand quartier musulman du même nom et bloquée à l'est par la falaise du Muqattam. Elle est quasi introuvable, sauf à suivre les camionnettes des zabbâlin par l'unique route qui y mène. La distinction religieuse s'y révèle par les reproductions d'icônes dans les ateliers et les fresques peintes sur les façades tandis que sur les quelques maisons musulmanes, on lit des versets du Coran; le quartier comprend aussi le lieu de pèlerinage dédié à Saint Samâan, "le tanneur ". Les zabbâlin de Manchiat Nasser bénéficient des nombreux liens avec la grande bourgeoisie chrétienne du Caire et des $œ u v r e s$ caritatives, telle celle de Sœur Emmanuelle. Construit en dur et administrativement reconnu au début des années 1990 même si non régularisé, les conditions de vie y sont meilleures que dans les autres petites zarâyeb en raison des très nombreux ateliers de recyclage et porcheries. A Batn al-Ba'ara, littéralement le Ventre de la Vache, la pauvreté et la précarité sont extrêmes: il s'agit de l'un des plus anciens quartiers de zabbâlin (1940), situé dans la cuvette d'une ancienne carrière et aujourd'hui encerclé par des immeubles en construction. Le quartier, qui ne dispose d'aucune sécurité foncière et qui se situe non loin d'un projet présidentiel de musée, est menacé d'une démolition imminente. Les coptes et musulmans y collectent et trient les déchets, revendus à Manchiat Nasser pour le recyclage. Ils élevaient, vendaient et consommaient des cochons, leur principale source de richesse avant leur abattage en mai 2009. L'exclamation sans doute la plus emblématique des effets de la crise du cochon nous a été livrée par un habitant musulman de Batn al-Ba'ara : "Que Dieu protège nos cochons ! "... Les zarâyeb situées sur des terrains agricoles, à l'instar de 'Izbat al-Nakhl' (1960), 'Ard el-Lioua (1969) et el-Baragil (1970) bénéficient d'une sorte de " protection foncière " du fait que les zabbâlin y ont acheté leurs terrains, même si la construction sur terres agricoles est illégale. Néanmoins, les déguerpissements précédents, souvent violents, et les ambitions urbaines et immobilières à venir, notamment celles qui concernent Manchiat Nasser dans le cadre du projet "Grand Caire 2050 " montrent que leur présence en ville reste très menacée. Le quartier de Tourah (1975), environ 7000 personnes dont $50 \%$ de confession musulmane, dispose d'un statut un peu particulier puisque, grâce à I'Association pour la Protection de l'Environnement (APE), cet ancien bidonville a été démoli en 1989, puis reconstruit sous forme d'immeubles sur des terrains vendus par le gouvernorat du Caire tandis que les porcheries et, en partie, le tri ont été délocalisées sur la décharge de Qattamiyya (Faccini, 1999, p. 22). Enfin, un petit campement de zabbâlin, d'une centaine de familles s'est installé " dans la montagne ", sans eau ni électricité, à proximité de la ville nouvelle de 15 de Mai.
4 - Une vidéo mise en ligne par l'association Mahaba montre assez bien l'aspect de 'Izbat alNakhl et de Manchiat Nasser, en dépit d'une musique tantôt dramatisante tantôt

grandiloquente :

http://chiffonniersducaire.overblog.com/article29126127.html

Figure 1 : Les Zabbâlin au Caire - 2010

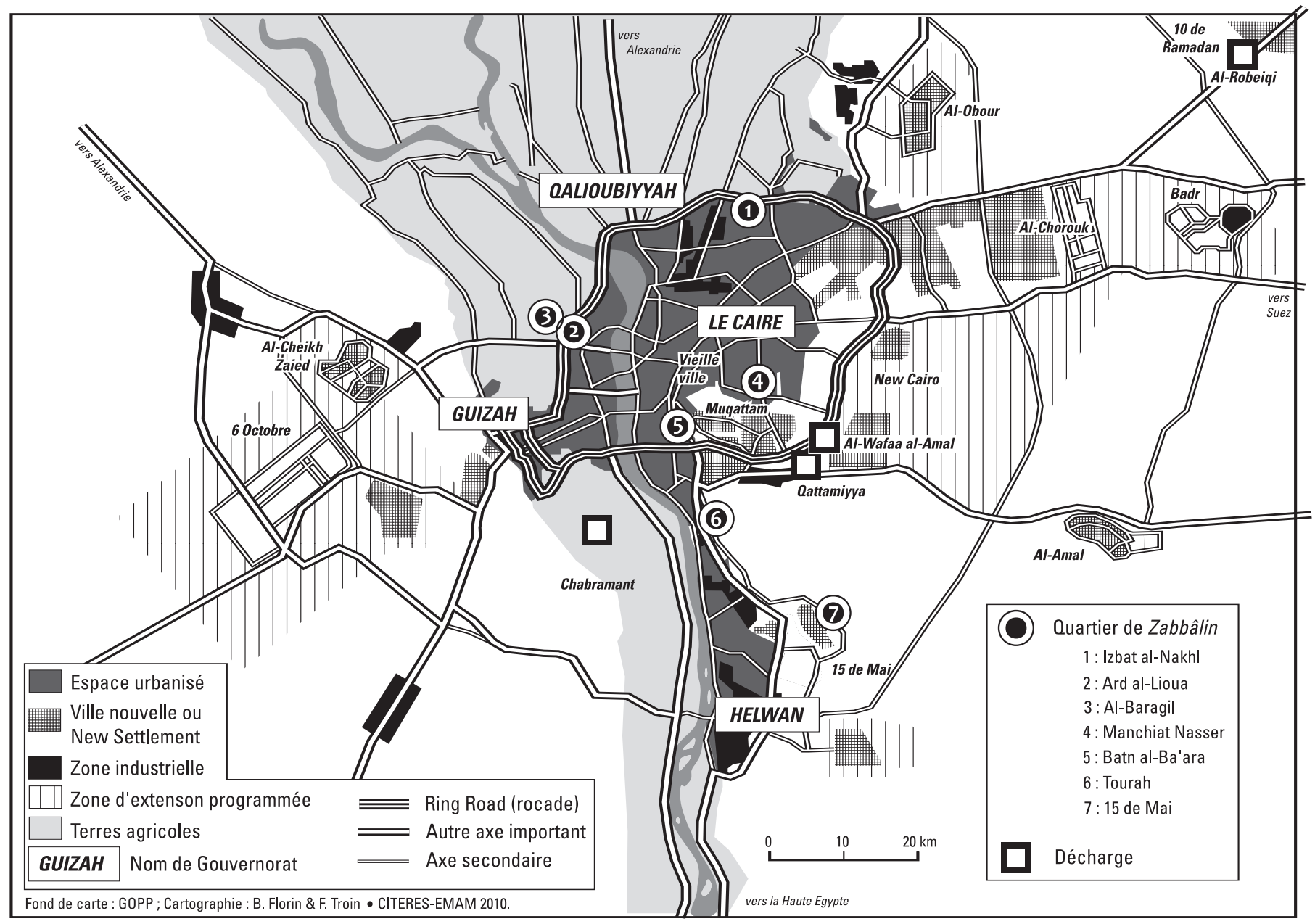


5 - Les wâhiya constituent aussi une corporation fondée sur l'origine géographique, les réseaux de parenté et un quartier commun Bâb al-Bahr, dans la vieille ville, où ils fréquentent les cafés de la rue Clot-Bey. Leur association professionnelle, enregistrée au ministère des Affaires

Sociales, gère les conflits de la communauté et les représente vis-à-vis des autorités (Assaad, 1988, p. 186-187). Par ailleurs, dans son roman Ahlam et les éboueurs du Caire, Fawzia Assaad relate avec justesse les relations entre oasiens et zabbâlîn (Assaad, 2006). L'organisation corporatiste des zabbâlin :
complémentarité et hiérarchie

L'organisation communautaire des zabbâlin se conjugue nécessairement à l'organisation professionnelle, mais renvoie aussi à la stigmatisation dont ils sont l'objet (Dollet, 2003, p. 72). Le premier facteur d'unité et d'identité de la communauté est d'ordre religieux puisque la majorité des zabbâlin est copte - minorité religieuse constituant $10 \%$ de la population égyptienne. Leur origine géographique, à savoir la Haute-Egypte - le Sa'îd - constitue une autre référence culturelle et identitaire qui dessine des réseaux professionnels et familiaux précis, reliant les villages du Sa'îd aux zarâyeb du Caire. Le troisième facteur, le plus prégnant, réside dans leur activité même : Ia promiscuité avec les cochons - symbole de l'impur dans le monde musulman -, la saleté et l'odeur des déchets et le qualificatif même de "zabbâl " (poubelle, ordure), etc., sont autant d'éléments qui contribuent au rejet et expliquent aussi la discrétion de leur présence en ville lorsqu'ils travaillent. A ceci s'ajoute la marginalisation spatiale : situées à la lisière de la ville ou dans ses interstices, les zarâyeb sont des espaces invisibles et inconnus de la plupart des Cairotes. Pour autant, le nombre de familles de zabbâlin n'est pas négligeable bien qu'impossible à chiffrer avec précision (mais l'absence de statistiques officielles relève aussi de cette invisibilisation) : en 2003, S. Dollet notait que ce nombre était sous-estimé par les autorités égyptiennes afin de minimiser leur rôle, mais qu'il était surévalué par les zabbâlin eux-mêmes ou par les ONG qui ont pris leur défense lors de la « crise " des années 2000. En recoupant les données, on peut penser qu'ils sont environ 100000 , dont environ 40000 à Manchiat Nasser et 8000 à 'Izbat al-Nakhl, les deux plus grands quartiers (encadré et fig. 1).

L'organisation communautaire, renforcée par l'endogamie, explique les liens professionnels et les complémentarités des activités à l'intérieur d'une même zerîba et entre les zarâyeb. Ainsi, par le jeu des alliances de parenté, une famille élargie peut effectuer l'ensemble de la chaîne, depuis la collecte des poubelles jusqu'au produit fini, en passant par les étapes de tri, de recyclage et d'élevage des porcs; ou, au contraire, être spécialisée dans une seule activité. Les interconnaissances, familiales et de voisinage, constituent des réseaux professionnels qui renforcent l'efficacité du système d'achat/vente des " matières premières " et, parfois même, du troc entre les familles. Les échanges entre les zarâyeb les rendent, pour certaines activités, complémentaires et dépendantes les unes des autres: c'est à Manchiat Nasser, par exemple, qu'il y a le plus d'ateliers (environ 700) et de machines-outils pour le recyclage, d'où les va-et- vient entre quartiers pourtant éloignés les uns des autres.

Sans doute, avant la réforme, peut-on parler d'une réelle corporation fondée sur un système communautaire structurant I'organisation professionnelle, elle-même réglementée par des accords, le plus souvent oraux: le fait que les territoires de collecte sont très précisément définis et répartis entre eux, sans empiètements territoriaux, ou que le parcours d'un zabbâl est transmis à ses héritiers, ou encore qu'il existe un marché des parcours en fonction de la richesse des quartiers relèvent de ces alliances et accords professionnels qui règlent les activités de collecte et de recyclage et qui déterminent les circulations de marchandises et d'argent au sein des quartiers et entre eux.

Cette organisation n'est pas contradictoire, loin s'en faut, avec une hiérarchie professionnelle marquée et, partant, avec une grande inégalité des revenus et des positions sociales à l'intérieur des quartiers et entre ces derniers. La " richesse " des familles dépend de l'ancienneté de l'ancrage résidentiel et professionnel, du nombre de véhicules de ramassage, de la nature des matériaux recyclés, de la valeur des machines servant au recyclage, du nombre de personnes qui est employé à la collecte, au tri, au recyclage ou aux relations commerciales avec l'extérieur activités qui ne dégagent pas les mêmes bénéfices et qui ne renvoient pas aux mêmes positions sociales. Au sommet de la hiérarchie se trouvent les patrons, propriétaires d'ateliers de recyclage, disposant de camions de ramassage, employant plusieurs familles et en lien avec les entreprises du secteur formel. Les écarts de revenus sont flagrants lorsque I'on voit les habitations de plusieurs étages en dur et plutôt bien aménagées de Manchiat Nasser et les bicoques de Batn al-Ba'ara ou de 'lzbat al-Nakhl. La différenciation est encore accentuée par la focalisation des associations et des aides sur Manchiat Nasser, et, dans une moindre mesure, sur Tourah et 'Izbat al- Nakhl. Les réformes des années 1980 et 2000 souligneront davantage ces inégalités et reconfigureront en partie les hiérarchies professionnelles.

\section{Le tri, le recyclage et les circulations professionnelles}

Les données sur l'activité de collecte des déchets ménagers par les zabbâlin sont variables, simplement parce que leur activité globale est impossible à quantifier. Les chiffres émanant des responsables politiques, retransmis par la presse, paraissent sous-estimer leur travail afin de légitimer les réformes. De même, les chiffres sur la production de déchets par le Grand Caire varient entre 10000 et 16000 tonnes par jour 
mais, sur les trois décharges du Grand Caire, celle de Wafaa al-'Amal accueillerait à elle seule 12000 tonnes. D'après les associations, les zabbâlin collecteraient entre 3000 et 5000 tonnes, essentiellement dans les quartiers de classes moyennes et aisées, ce qui signifie bien sûr que la couverture de la ville $n^{\prime}$ est que partielle ${ }^{6}$ : la collecte dans les villes nouvelles est effectuée par des entreprises spécifiques et, dans les quartiers populaires, de grandes bennes sont irrégulièrement vidées par les services municipaux. Les associations s'accordent sur la capacité de recyclage des zabbâlin qui s'élèverait à $80 \%$ des déchets ramassés, I'un des meilleurs taux au monde, chiffre sous-estimé, à nouveau, par le ministère de l'Environnement qui l'évalue à $30 \%$ (Dollet, 2003, p. 33). Bien davantage que la collecte, le recyclage et, avant mai 2009, l'élevage des porcs représentent la principale source de revenus des familles: d'après le président de I'Association of Garbage Collectors' ${ }^{7}$, le salaire moyen d'un zabbâl est d'un peu plus de 500 Livres égyptiennes ( $\mathrm{fe}$ ) en 2008 - 62 Euros - tandis que le salaire moyen en Egypte s'établit à environ 300 fe par mois.

Les matières organiques, $40 \%$ de la collecte, alimentent les cochons noirs des zarâyeb dont la viande est vendue à quatre grands commerçants qui détiennent le monopole de la revente; le lisier, quant à lui, sert à la fabrication très rentable d'un compost de qualité. Les autres matériaux sont triés par les femmes et les enfants, en fonction de leur composition (cuivre, aluminium, métal, verre, plastique, tissu, papier, etc.) et la classification s'opère de façon très fine, notamment en ce qui concerne la récupération des plastiques. La rentabilité du recyclage, liée à la "richesse " initiale du matériau et à la valeur du produit qui en sera issu, établit une sorte de hiérarchie des déchets et donc la hiérarchie entre les zabbâlin. Les circulations entre les ateliers de recyclage et le secteur formel sont nombreuses: les matériaux triés et/ou recyclés (carton, métaux plastiques, etc.) peuvent être vendus aux usines, en particulier celles des villes nouvelles de Dix de Ramadan et de Six Octobre ; certains produits finis sont livrés en centre-ville aux commerces et grossistes; d'autres produits empruntent des circuits plus longs à l'instar d'une partie du plastique PET (polyéthylènetéréphtal) qui est exportée à hauteur de $3000 \mathrm{fe}$ la tonne en Chine, pays avec lequel les échanges sont de plus en plus importants à partir des années 2000.

Ainsi, loin d'être isolée ou repliée sur elle-même, I'activité des zabbâlin s'inscrit dans un réseau de relations: les wâhiya, les habitants du Caire dont ils collectent les poubelles, les entreprises du secteur formel, les grossistes et commerces ; quant aux parcours de collecte et aux échanges entre zarâyeb, entre les zarâyeb et les villes nouvelles ou encore à l'intérieur des quartiers, ils forment des circulations et des territoires à la fois précis et régis par les accords noués au sein de la communauté, mais ils sont aussi adaptables en fonction des contraintes ou des opportunités de l'activité.

\section{LES RÉFORMES ET LA RECONFIGURATION DES TERRITOIRES PROFESSIONNELS: DES COMPÉTENCES À L'AJUSTEMENT INÉGALES}

\section{Les réformes des années 1980 : la privatisation partielle du secteur informel}

Dans la ligne de la rhétorique modernisatrice qui avait présidé à la décision de créer les villes nouvelles (Florin, 2005), les années 1980 sont celles des déclarations, énoncées haut et fort par les gouverneurs successifs, sur la nécessité de moderniser la capitale afin de lui donner - ou lui rendre, c'est selon - son statut de grande ville du monde arabe : les villes nouvelles, la construction de la rocade et du métro, les annonces récurrentes de la fermeture du Caire à l'immigration rurale pourtant devenue résiduelle, ou les projets de démolition des quartiers informels "trop visibles" plutôt que la régularisation et la réhabilitation (Séjourné, 2006, p. 459) participent de la même idéologie réformatrice. Dans cette perspective les Cairo Cleaning and Beautification Agency (CCBA) et Guizah Cleaning and Beautification Agency (GCBA) sont instituées en 1983, qui ont la charge de rendre efficients les plans précédents de propreté des espaces publics.

En ce qui concerne les déchets des particuliers, le changement est substantiel puisque la collecte doit, dès lors, être effectuée par des entreprises détentrices d'une licence qu'elles achètent aux CCBA et GCBA: il s'agit bien ici de la formalisation - dans une logique de contrôle d'un segment de l'activité des zabbâlin et des wâhiya, celle du ramassage. Et, en effet, une soixantaine $d^{\prime}$ entreprises, associant parfois les deux corporations demandent et obtiennent une licence (Debout 2007, p. 20). Le paradoxe réside ici dans le fait que la régularisation, certes contrainte et qui ne donne accès à aucun droit spécifique, constitue une reconnaissance officielle de la collecte par les "entreprises " des zabbâlin alors que les activités de tri et de recyclage ressortissent toujours du secteur informel; ceci signifie encore que toute collecte effectuée en dehors du cadre des entreprises devient non plus "informelle " mais illégale. Last but not least, I'interdiction faite à toute carriole tractée par des ânes de pénétrer en ville, si elle n'a pas concerné que les zabbâlin loin s'en faut et si elle a mis un temps certain à être appliquée, a imposé aux ramasseurs qui en avaient les moyens l'achat de véhicules (pick-up ou camions) en remplacement des charrettes. De ces décisions découle I'accentuation des inégalités et des rapports de dépendance entre zabbâlin puisque ceux qui n'ont pas eu les moyens d'acheter la licence ou un véhicule motorisé $n^{\prime}$ ont plus, en théorie, un accès
6 - Il existe des « territoires de la récupération " précis qui ne se superposent pas nécessairement à la densité de population, mais qui s'établissent en fonction du niveau socio-économique des habitants puisque " plus la production de déchets par personne et par jour est élevée, plus la production par quartier est importante. La distribution pondérale des déchets recyclables est bien fonction d'un niveau supérieur de consommation " (Carré, 2008, p. 2). A titre d'exemple, le quartier aisé de Maâdi, au sud de la capitale, a représenté un enjeu de taille pour les zabbâlin, lorsque en 2002 il a été attribué à une société privée égyptienne Europa 2000.

7 - Cette association, créée en 1974 et basée à Manchiat Nasser, compte une centaine de membres. Elle est I'un des interlocuteurs reconnus des gouvernement et gouvernorat. 
8 - Titre de l'article de Marwa Hussein dans al-Ahram Hebdo du 27 août 2003.

9 - Lise Debout évoque l'injustice de ce mode de paiement : ainsi un boucher qui génère beaucoup d'ordures mais consomme peu d'électricité payera moins qu'un bijoutier qui consomme davantage d'électricité mais produit peu de déchets (Debout, 2007, p. 32)

Aujourd'hui, les Cairotes paient le service de collecte sur leur facture d'électricité, en fonction de trois catégories les habitants des quartiers " chics " versent 10 fe par mois, ceux des quartiers "moyens " $5 \mathrm{fe}$, ceux des quartiers populaires $3 \mathrm{fe}$ en plus de ce qui est payé aux zabbâlin.

\section{0 - Face à l'ampleur}

inattendue de la contestation et instrumentalisant le registre nationaliste, les gouvernorats ont mis en place en 2003 des " lignes téléphoniques rouges " qui enregistrent les plaintes des " citoyens mécontents du travail des sociétés étrangères " (al-Wafd, 15/04/03) direct aux déchets. Cependant, il faut relativiser cette dernière assertion par le fait que certains d'entre eux ont adopté d'autres stratégies professionnelles, notamment en développant uniquement l'activité de recyclage, plus valorisante financièrement et symboliquement que celle de collecte; d'autres louent les véhicules de familles voisines ou les payent au chargement, d'autres encore contournent ces contraintes.

\section{"Recyclage difficile pour les chiffonniers ${ }^{8}:$ les conséquences de la délégation de la gestion des déchets aux sociétés privées}

La décision de déléguer au secteur privé la gestion des déchets est prise en 1999 par le gouvernement égyptien et, en septembre 2000, le gouvernorat d'Alexandrie ouvre le ban en signant un contrat avec Onyx, filiale de Véolia Environnement. En 2002, le gouvernorat du Caire (est du Nil) est divisé en quatre zones et le gouvernorat de Guizah (ouest du Nil) en deux zones: des appels d'offre, spécifiques pour chacun de ces six espaces, sont publiés dans la presse. Certains contrats imposent, par exemple, un critère d'attribution des marchés qui, de facto, exclura toute candidature possible de la part d'une entreprise égyptienne, publique ou privée: ainsi, est-il demandé aux entreprises d'avoir une expérience de gestion des déchets d'au moins vingt ans et dans une grande ville (Dollet, 2003, p. $39)$; or, en Egypte, le système informel prévaut et les entreprises publiques de nettoyage des rues (Europa 2000, Misr Service, Cairo Service ou Hayyît al-Nadafa) sont de création récente et ne sont pas formées à la collecte. Dans d'autres contrats, les sociétés nationales peuvent être candidates mais, ainsi que l'explique Lise Debout, elles ne seront pas retenues en raison de leurs moyens techniques inférieurs à ceux offerts par les sociétés étrangères ou de leur coût supérieur. En définitive, les sociétés retenues sont les deux italiennes Ama Arab (responsable de la gestion des déchets de Rome) pour le nord du Caire et IES du groupe Lacorossi/Genesu pour le nord de Guizah, trois sociétés espagnoles (ENSER/Urbaser, EES/FCC et IES/FCC-Vivendi) respectivement chargées du Caire ouest, du Caire est et du sud de Guizah (Debout, 2007, p. 30-31). Le sud du Caire, seule zone qui n'était pas comprise dans les appels d'offre, est en 2003 finalement alloué à l'entreprise privée égyptienne Europa 2000 et à l'entreprise publique égyptienne Fûstat. Ces entreprises doivent effectuer la collecte des déchets ménagers grâce à des conteneurs achetés par les habitants, assurer le nettoyage des rues, mais aussi sensibiliser la population ; elles sont supposées recycler $20 \%$ de leur collecte et enfouir le reste dans des décharges du désert (al-Ahram, 12 mars 2003). Aucune clause ne stipule qu'elles doivent employer des zabbâlin et rien n'est dit, à aucun moment, sur le système antérieur de collecte et de recyclage. Dans la même logique, ni les zabbâlin, ni leurs associations ne seront avertis de la réforme puisqu'ils apprennent la nouvelle par la presse...

Les vives réactions des Cairotes contre le nouveau système, relayées par les médias qui prennent parti contre les "sociétés étrangères", ont pour effet de mettre au grand jour le rôle des zabbâlin. Cette improbable alliance s'établit lorsque se multiplient les plaintes d'habitants et commerçants, faisant référence à l'ancien système des zabbâlin, moins coûteux et plus efficace à leurs yeux: intégrée aux factures d'électricité, la nouvelle taxe de collecte s'établit en fonction de la consommation électrique, du type de bâtiment et de son activitég. Au début de I'année 2004, la cessation du paiement des factures dans les quartiers concernés par le ramassage pose problème aux gouvernorats qui, escomptant ces revenus, n'honorent pas les contrats les liant aux sociétés européennes. En juin 2005, les tensions s'avivent entre le gouvernorat du Caire et la société espagnole Enser (filiale d'Urbaser) qui suspend la collecte des déchets et ne verse plus les salaires de ses employés égyptiens - ce qui provoque des manifestations de rue de la part de ces derniers. Les ordures s'accumulent, l'odeur est insoutenable, d'autant qu'il fait de plus en plus chaud... Les amendes imposées par le gouvernorat à la société espagnole pour travail non fait s'élèvent à 100 millions de $\mathrm{fe}^{10}$; les deux parties s'intentent mutuellement un procès fleuve et sans verdict qui s'achève par un recours à l'arbitrage international et par le départ d'Enser (al-Gûmhuriya, 17 mai 2006), remplacée par la suite par Ama Arab (italienne).

La crise, qui a impliqué des acteurs auss différents que les multinationales, les Cairotes, le ministère de l'Environnement opposé à celui de I'Energie, a mis sur le devant de la scène les zabbâlin: des témoignages d'habitants sont publiés dans la presse évoquant les liens indéfectibles qui lient les Cairotes à "leur " zabbâl et l'efficacité de la collecte au porte-à-porte. À cette défense directe des zabbâlin, s'ajoute un registre d'ordre culturel et nationaliste: les Egyptiens ne peuvent s'adapter aussi brutalement à un "changement de leurs habitudes ", d'autant plus si celui-ci est imposé par des sociétés étrangères aux " traditions locales ". Le sentiment de trahison par les autorités politiques est aussi récurrent : pourquoi le gouvernement n'a-t-il pas choisi des sociétés égyptiennes? Ou, tout au moins, conservé l'ancien système puisque " une cohorte de 100000 éboueurs effectuait chaque jour un ingénieux ramassage de 6000 tonnes d'ordures qui ne coûtait au contribuable qu'une minime aumône mensuelle octroyée aux déguenillés qui escaladaient les étages de nos immeubles (al-Ahram Hebdo, 6/07/05) ? En définitive "ce sont les Cairotes qui sont devenus des zabbâlin pour les sociétés étrangères qui ne font pas leur travail » (al-Gûmhuriya, 17/05/06) ... 
Pour les zabbâlin, la réforme annule, du jour au lendemain, la régularisation de leur activité certes partielle puisque ne concernant que la collecte - qui leur avait été concédée par les licences. De plus, ils ne peuvent plus a priori réclamer d'argent aux habitants qui payent dorénavant par ailleurs. Enfin, et surtout, ils n'ont plus, a priori encore, accès légalement aux déchets devenus propriété des sociétés privées. Tout ceci signifie dans l'absolu la fin des porcheries et du recyclage. La mobilisation, qui échoue, puis les différentes stratégies d'adaptation ou de contournement qu'ils mettent en œuvre témoignent des capacités, inégales, à l'ajustement tant de leurs pratiques que de leurs territoires professionnels.

\section{NOUVELLES PRATIQUES ET RECONFIGURATIONS DES TERRITOIRES PROFESSIONNELS DES ZABBÂLIN} Oppositions versus arrangements : la
formalisation de l'informel?

La résistance des zabbâlin face à la perte de leurs territoires professionnels se traduit tout d'abord par des manifestations, le 3 février 2003, à 'Ard elLioua et Manchiat Nasser où ils occupent I'autoroute Salâh Sallem qui passe en contrebas du quartier; les forces de l'ordre interviennent assez violemment et quatre personnes sont arrêtées (Dollet, 2003, p. 114-133). L'intériorisation du caractère éminemment autoritaire de l'Etat, alors que l'état d'urgence est toujours en vigueur, est sans doute I'un des facteurs expliquant les limites de la mobilisation collective des zabbâlin. Le sentiment de marginalisation de ces derniers a aussi renforcé la crainte d'une expression publique trop forte - d'ailleurs, les espaces de manifestation se situant à proximité des zarâyeb, et non en centre-ville, leur visibilité fut plutôt réduite. Parallèlement, les " petites" oppositions individuelles des zabbâlin se sont multipliées, relatées par la presse: dégradation ou vol des conteneurs, éparpillement des déchets des bennes dans les rues et multiplication des plaintes sur les "lignes rouges" du gouvernorat contre les sociétés étrangères qui étaient alors astreintes à des amendes pour travail non fait...

La réforme suscite aussi des clivages au sein de la communauté, mettant en exergue les inégalités entre zabbâlin, mais aussi entre les quartiers: Manchiat Nasser focalise I'attention de la presse et des débats alors que les petites zarâyeb sont tenues à l'écart du processus de négociation (Dollet, 2003, p. 121). En définitive, c'est par le biais des interrelations et des réseaux de pouvoir, politiques et associatifs, que l'ajustement à la contrainte s'effectue. Hormis les religieux, silencieux en dehors de la communauté, les responsables $d$ 'associations et députés mènent les transactions "officielles ". Le quotidien al-
Ahram du 12 mars 2003 relate ainsi les propos tenus par Mohamad Bahdadi, député de Manchiat Nasser, face à l'Assemblée nationale, au gouverneur du Caire et au ministre de I'Environnement : arguant du fait que les zabbâlin sont 30000 dans sa circonscription et que "leur métier, c'est les poubelles ", le député les présente comme les premières victimes de la "privatisation " et demande la rupture des contrats avec les sociétés privées pour le sud du Caire qui serait laissé aux zabbâlin; il réclame encore que ceux-ci aient le droit de ramasser les déchets que les sociétés enterrent dans les décharges. Le gouverneur lui répond que le problème des zabbâlin est résolu puisqu'ils pourront être embauchés pour 250 fe par mois par les sociétés étrangères. Quant au ministre de I'Environnement, Mamdouh Riyad, il assure: "Mon cœur est avec les zabbâlin de Manchiat Nasser (...) mais il est impossible de retourner à la situation antérieure. Regardez Alexandrie qui est un bon exemple du succès des sociétés étrangères : c'est un transfert de civilisation (...). II faut profiter des techniques de ces sociétés pour fonder des entreprises nationales et leur faire concurrence ". Le représentant des zabbâlin, Rafik Assaad, rétorque que cette concurrence lui semble impossible en raison d'un "décalage de plusieurs années de civilisation "entre les zabbâlin et les sociétés étrangères, qui sont solidaires et dont les techniques sont plus performantes; il achève son propos par: "Je suis contre ces sociétés étrangères et contre ces contrats qui ont été conclus!».

Ces débats ouvrent les transactions entre les représentants du gouvernorat, des sociétés européennes et les zabbâlin influents, à savoir les responsables d'associations et les patrons d'ateliers. La proposition des zabbâlin de constituer une société unique - ce qui aurait supposé une organisation complexe au sein de leur communauté - est immédiatement rejetée par les responsables. En revanche, ces derniers leur proposent de se faire embaucher comme éboueurs ou balayeurs par les sociétés, sous certaines conditions ${ }^{11}$ et, surtout, par signer de contrats de sous-traitance pour la collecte. Si les patrons "bien placés " ont effectivement signé des contrats, de nouvelles sociétés intermédiaires égyptiennes se sont aussi créées opportunément comme la société Rose Service, rétribuée 2 fe par appartement par FCC et qui emploie en soustraitance des zabbâlin eux-mêmes payés 1 fe par logement (al-Wafd, janvier 2007). D'autres zabbâlin ont conclu des "permis de tri " pour avoir le droit de récupérer sur les décharges périphériques des déchets à recycler qu'ils ramènent au Caire...

Si les contrats de sous-traitance amorcent une formalisation partielle du secteur informel de la collecte, les transactions ont néanmoins exclu les 

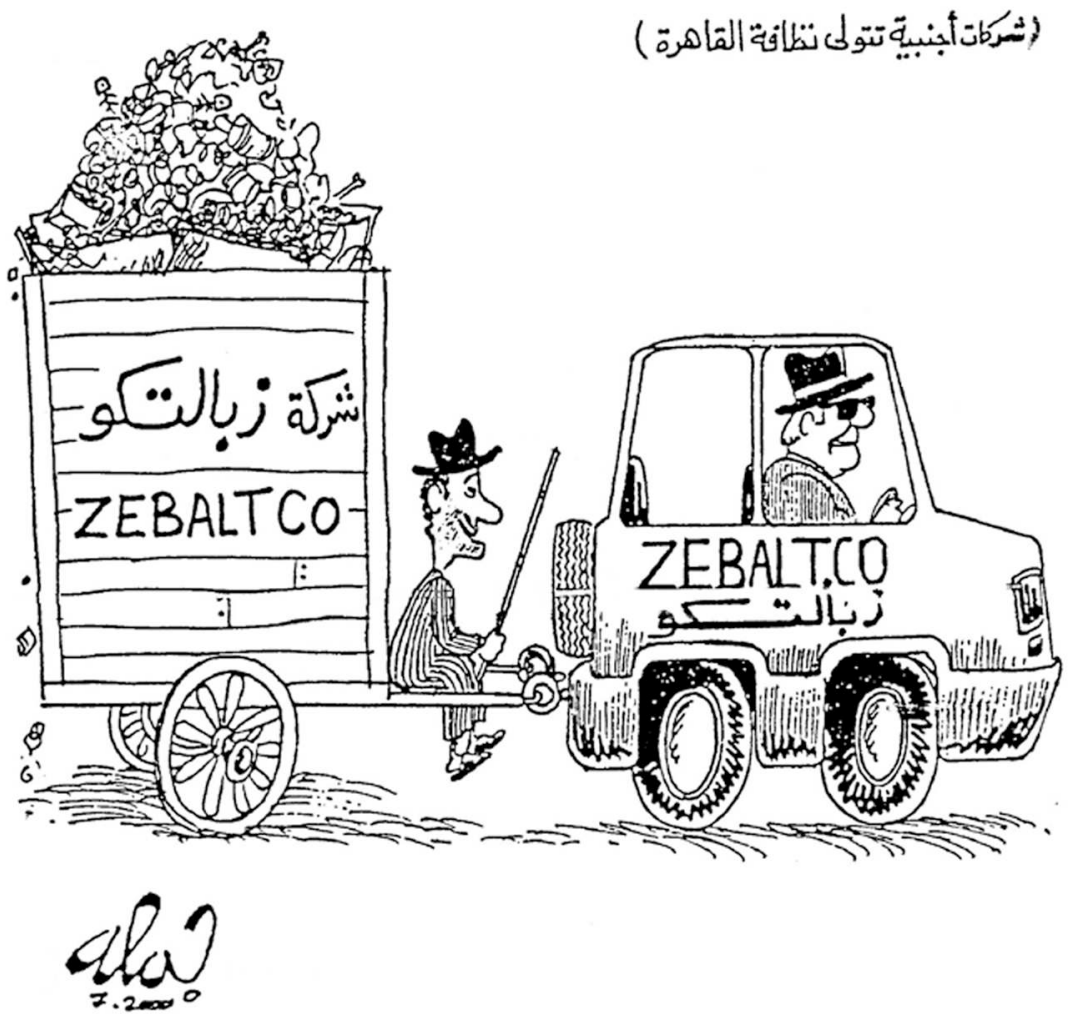

11 - Ces conditions sont : être un homme, avoir entre 18 et 40 ans, être dégagé des obligations militaires, savoir lire et écrire, ne pas être malade.

Or, de nombreux zabbâlin sont analphabètes et ont des maladies chroniques (Dollet, 2003, p. 138). Par ailleurs, les salaires proposés sont moitié moins élevés que le salaire moyen d'un zabbâl: " Le gouvernorat de Guizah a vendu ses zabbâlin aux sociétés étrangères " titre le journal alMidan du $1^{\text {er }}$ mars 2004.

D'après nos entretiens, aucun zabbâl de Manchiat Nasser, de 'Ard al-Lioua ou Batn al-Ba'ara n'aurait accepté d'être embauché.

12 - Pierre Desvaux utilise le terme anglais "scavengers" "pilleurs de poubelles" (Robert \& Collins). Le mot égyptien est " sahîr " que l'on peut traduire par " vagabond" (Desvaux, 2009, p. 64). Le terme français " biffin " me paraît plus à même de qualifier ces ramasseurs dont la collecte est aléatoire, effectuée à pied, avec un sac porté au dos, ramené une fois rempli dans la zeriba.

Contrairement à l'ancienne forme de collecte qui reposait sur le tri dans la zérîba, les biffins sélectionnent les déchets sur place et remettent (plus ou moins) ceux qui ne les intéressent pas dans les bennes. zabbâlin les moins bien "placés" dans la qui vivent dans les petites zarâyeb isolées. Pour ces derniers, les stratégies de contournement ne peuvent passer par des arrangements légaux.

Voir caricature "les entreprises étrangères prennent en main la propreté du Caire " (fig. 2)

\section{"Le chat et la souris ": de l'informalité à I'illégalité}

Les stratégies de contournement de la contrainte consistent, comme l'explique le zabbâl 'Atef, à jouer au chat et à la souris avec les autorités et les camions bennes des compagnies, ce qui implique une modification complète des temporalités de travail mais aussi des circuits de collecte: "Nos horaires ont changé, nous passons à 3 heures du matin et quand ceux de la société arrivent, il n'y a plus grand chose à prendre... I/s sont très contents car, comme ça, ils n'ont rien à faire!". Les " tournées " s'effectuent donc la nuit et les parcours varient afin d'échapper à la confiscation du matériel de collecte, aux amendes et aux réprimandes. Certes, I'activité de ces zabbâlin est devenue illégale, mais leurs façons de faire sont très diverses, révélant l'inégalité des positions et des capacités d'adaptation à la contrainte. La hiérarchie professionnelle, différente de ce qui prévalait dans le système antérieur, semble ainsi s'être accentuée entre les propriétaires de camions qui ont conclu des accords de soustraitance et les "biffins ${ }^{12}$, nouvelle catégorie de ramasseurs, jusque là inexistante au Caire : ces derniers, nouveaux venus ou anciens zabbâlin exclus du système légal, sont dans une situation particulièrement précaire. Très exposés au risque d'arrestation, ils entrent en concurrence avec les zabbâlin mieux lotis qui disposent d'un pick-up ou qui travaillent pour une société. Entre les mieux et les moins bien placés, l'éventail de manières de procéder complète cette hiérarchie professionnelle : certains zabbâlin ont passé des communauté pour avoir voix au chapitre ou ceux
Figure 2: "Les entreprises

étrangères prennent en main la propreté du Caire "

sources : Al-Ahram,

16 novembre 2002 in Soazig

Dollet, 2003, p. 43

accords avec les commerçants qui les rétribuent afin d'enlever les déchets, d'autres se sont arrangés avec les concierges pour avoir accès aux déchets, d'autres encore collectent, au porte à porte, les déchets de leurs anciens clients, $d^{\prime}$ autres se sont spécialisés dans la collecte d'un type de déchet, d'autres, assez nombreux semble$\mathrm{t}$-il, trient les déchets recyclables des décharges publiques - en échange de pots de vins distribués aux gardiens - ...

Dans certains quartiers, la superposition des territoires professionnels, qui avant la réforme étaient bien distincts, est de mise ainsi que I'explique Ahmad, habitant de Zaytûn : "Dans mon quartier, je les vois tous passer: le plus pauvre qui a juste son sac, celui qui a une charrette et un âne (interdits en ville), ceux qui ont des camionnettes et puis enfin, les camions des sociétés, mais, à ce moment là, il n'y a plus rien à prendre, les autres ont déjà tout ramassé !". Pour autant, dans les quartiers aisés, la compétition rejette les moins bien placés dans la hiérarchie professionnelle qui sont, à l'inverse, tolérés dans les quartiers populaires (Desvaux, 2009, p. 74). Enfin, un certain nombre de familles - impossible à chiffrer - n'a pu que difficilement, ou parfois pas du tout, s'adapter à la contrainte. C'est, par exemple, le cas de celles qui ont retiré leurs enfants de l'école pour épargner le salaire d'un employé ; ou d'autres qui, d'après 'Atef, ne pouvant plus vivre au Caire, seraient retournées en Haute-Egypte; ou encore, la reconversion obligée des wâhiya traditionnels... Quoi qu'il en soit, le sentiment d'avoir été exclu du processus et l'amertume qui en découle sont très prégnants comme l'exprime encore 'Atef: "On fait tout le boulot à la place des sociétés étrangères puisqu'elles passent après nous et qu'il n'y a presque plus rien à ramasser et c'est elles qui sont payées à notre place ".

\section{CONCLUSION}

La réforme du système de gestion des déchets délégant à des sociétés européennes la collecte - car, de facto, elles n'effectuent pas le recyclage a eu comme effet majeur d'accentuer les écarts entre les zabbâlin, d'autant que les compétences à I'adaptation et les capacités à contourner la contrainte sont très inégales et liées aux positions déjà différenciées des uns et des autres avant la " crise " : I'apparition des biffins est probablement I'un des effets les plus évidents de la réforme et de cette nouvelle hiérarchie entre zabbâlin ; beaucoup plus invisibles sont les situations de dépendance, au sein de la communauté, qui touchent les femmes isolées (veuves, divorcées), les jeunes hommes ou encore les zabbâlin des petites zarâyeb. Dans le même ordre d'idées, la superposition des territoires professionnels qui, dans la configuration antérieure étaient distincts, révèle la complexité des façons de faire, mais 
aussi de nouvelles alliances ou concurrences, de la disparition et de I'apparition de nouveaux acteurs. La réforme témoigne aussi des relations paradoxales qu'entretiennent depuis longtemps les autorités publiques avec les zabbâlin: leur reconnaissance partielle, réservée à Manchiat Nasser, avait été impulsée quand les associations sont devenues des interlocutrices légitimes du pouvoir, à partir des années 1980; la régularisation de Manchiat Nasser, qui en a découlé, n'a pas empêché les menaces d'expulsion pour ce même quartier, décuplées pour les autres zarâyeb. Par la suite, l'imposition du système des licences de ramassage et l'organisation en entreprises de collecte a constitué un contrôle de leurs activités, mais pouvait être interprétée comme une légitimation de leur travail, infirmée brutalement par la "privatisation " des années 2000. Celle-ci est vécue comme la négation de leur existence, mais la mobilisation et la médiatisation qui suivent la " crise " imposent des débats, faute de droits, des transactions, puis la réinsertion, très relative, des zabbâlin dans le système formel privé. Relevant aussi de la complexité de la position de la communauté copte en Egypte, la fragilité et I'ambivalence des relations entre les zabbâlin et l'Etat se sont à nouveau matérialisées dans la nouvelle "crise " liée à la grippe H1N1, qualifiée abusivement de "grippe porcine ", qui a conduit à l'abattage en mai 2009 de 300000 porcs, suivi de heurts violents entre zabbalîn et forces de l'ordre. Bien davantage que la délégation du service de collecte aux entreprises privées à laquelle ils se sont tant bien que mal ajustés, cette "crise du cochon " est vécue par la plupart des zabbâlin comme un coup fatal, porté à leur existence même. Ceci n'a pas empêché pour autant le gouvernement égyptien d'admettre, fin 2009, que leur activité est indispensable au maintien de la propreté de la capitale...

\section{BIBLIOGRAPHIE}

ABU-LUGHOD J., 1971, Cairo. 1001 Years of the City Victorious, Princeton, New Jersey, Princeton University Press, 283 p.

ARNAUD J.-L., 1993, Le Caire. Mise en place d'une ville moderne, 1867-1907, thèse de doctorat d'études arabes et islamiques sous la dir. de A. Raymond et R. Ilbert, Université de Provence, Aixen-Provence, $524 \mathrm{p}$.

ASSAAD F., 2006, Ahlam et les éboueurs du Caire, Grolley, Edition de l'Hèbe, 389 p.

ASSAAD R., 1988, L'informel structuré. Les zabbâlin du Caire, Peuples Méditerranéens, n4142, Paris, p. 181-192.

BESANÇON J., 1958, Une banlieue du Caire: Héliopolis, Revue de géographie de Lyon, vol. 33, $n^{\circ} 2$, p. 119-151.
CARRÉ M.-N., 2008, Buenos Aires, ou les territoires de la récupération, Géoconfluences, Le développement durable, approches géographiques, $16 \mathrm{p}$.

CLERGET M., 1934, Le Caire. Etude de géographie urbaine et d'histoire économique, Thèse pour le Doctorat Es Lettres présentée à la Faculté des Lettres de I'Université de Paris, Le Caire, Tome II, $416 \mathrm{p}$.

DEBOUT L., 2007, La libéralisation/modernisation en Egypte. La possibilité d'une réforme de la gestion urbaine? Le cas pionnier de la privatisation de la collecte des ordures ménagères au Caire et son impact sur la gestion de la métropole, mémoire de Master II, sous la dir. de C. LEFEVRE, Institut Français d'Urbanisme, Lyon, $81 \mathrm{p}$.

DE SILGUY C., 1996, Histoire des hommes et de leurs ordures. Du Moyen Âge à nos jours, Paris, Le Cherche Midi éd., 226 p.

DESVAUX P., 2009, Les impacts de la délégation de la gestion des déchets au Caire sur la filière informelle des ordures, mémoire de Master I de géographie, Université de Tours, sous la dir. de B. FLORIN, $100 \mathrm{p}$.

DOLLET S., 2003, Une communauté traditionnelle face à la modernité. Le cas des zabbâlin du Caire, mémoire de DEA de science politique, sous la dir. de E. PICARD, Université d'Aix-Marseille III, 193 p.

EL KADI G., BEN NEFISSA S., 1999, Les associations: nouveaux acteurs de I'aménagement et de la gestion de l'urbain en Egypte?, in SIGNOLES P. et al., L'urbain dans le monde arabe, Paris, CNRS ed., p. 320, 338.

FACCINI B., 1999, Les chiffons se recyclent, une nouvelle trame de vie se tisse, Innovations, jeunesse $n^{\circ} 3$, UNESCO, A.P.E., Paris, 41 p.

FLORIN B., 2005, Vivre en parallèle? L'évolution des villes nouvelles du Grand Caire, Les Annales de la Recherche Urbaine, p. 97-105.

ILBERT R., 1996, Alexandrie, 1830-1930, Le Caire, IFAO, $886 \mathrm{p}$.

RAYMOND A., 1993, Le Caire, Paris, Fayard, 428 p.

SÉJOURNÉ M., 2006, Les politiques récentes de traitement des quartiers illégaux au Caire: nouveaux enjeux et configurations du système $d^{\prime}$ acteurs?, thèse de doctorat en géographie, sous la dir. de P. SIGNOLES, Université de Tours, 591 p.
Adresse de l'auteur:

Université de Tours, UMR 6173 CNRS, CITERES -

EMAM, Equipe Monde arabe et Méditerranée, 33, allée

Ferdinand de Lesseps, 37000

Tours. Tel :0247201317 